\title{
Spectral Hump Formation in Visible Region of Supercontinuum from Shaped Femtosecond Laser Filamentation in Fused Silica
}

\author{
Junwei Chang ${ }^{1}$, Dongwei Li ${ }^{1}$, Tingting $\mathrm{Xi}^{2}$, Lanzhi Zhang ${ }^{1}$ and Zuoqiang Hao ${ }^{1, *}$ \\ 1 Shandong Provincial Engineering and Technical Center of Light Manipulations \& Shandong Provincial Key \\ Laboratory of Optics and Photonic Device, School of Physics and Electronics, Shandong Normal University, \\ Jinan 250014, China; changjunwei@sdnu.edu.cn (J.C.); lidongwei@sdnu.edu.cn (D.L.); \\ lzzhang@sdnu.edu.cn (L.Z.) \\ 2 School of Physical Sciences, University of Chinese Academy of Sciences, Beijing 101407, China; \\ ttxi@ucas.ac.cn \\ * Correspondence: zqhao@sdnu.edu.cn
}

Citation: Chang, J.; Li, D.; Xi, T.; Zhang, L.; Hao, Z. Spectral Hump Formation in Visible Region of Supercontinuum from Shaped Femtosecond Laser Filamentation in Fused Silica. Photonics 2021, 8, 339. https://doi.org/10.3390/ photonics 8080339

Received: 19 July 2021

Accepted: 17 August 2021

Published: 19 August 2021

Publisher's Note: MDPI stays neutral with regard to jurisdictional claims in published maps and institutional affiliations.

Copyright: (c) 2021 by the authors. Licensee MDPI, Basel, Switzerland. This article is an open access article distributed under the terms and conditions of the Creative Commons Attribution (CC BY) license (https:// creativecommons.org/licenses/by/ $4.0 /)$.

\begin{abstract}
We investigate experimentally the local intensity control in the visible region of the supercontinuum (SC) generated from femtosecond laser filamentation in fused silica by using pulse shaping technology. Based on the genetic algorithm, we show that a distinct spectral hump at any preset wavelength can be formed in the blue-side extension. The local intensity control in the SC could improve the abilities of the SC applications.
\end{abstract}

Keywords: filamentation; supercontinuum; pulse shaping; spectral hump

\section{Introduction}

Since spectral broadening by focusing a high-power picosecond laser pulse into crystals and glasses was discovered by Alfano and Shapiro, supercontinuum (SC) source has attracted widespread attention due to the spectral range's spectacular ability to span from ultraviolet to mid-infrared [1,2]. The broadband SC source has found a variety of applications, such as spectroscopy [3,4], fluorescence microscopy [5], optical coherence tomography [6,7], and chemical sensing [8]. Therefore, SC generation has been extensively investigated in various optical media under a wide variety of experimental conditions, including liquid, gas, and solid [9]. In the past decade, along with the development of photonic crystal and microstructured fibers, and highly nonlinear materials, the study of SC generation in optical fiber has achieved remarkable progress [10-15]. The SC with different broad spectra has been obtained. Diouf and colleagues obtained a super-flat coherent mid-infrared SC spectrum by using a chalcogenide photonic crystal fiber [13]. Adamu et al. reported the multi-octave SC spectrum spanning from deep ultraviolet $(200 \mathrm{~nm})$ to the mid-infrared $(4000 \mathrm{~nm})$ generation in a gas-filled hollow-core antiresonant fiber [14]. Furthermore, they demonstrated the mid-infrared SC with a spectrum spanning from $1.4 \mu \mathrm{m}$ to $13.3 \mu \mathrm{m}$ by using an ultra-high numerical aperture chalcogenide step-index fiber [15]. Today, a series of optical fiber-based commercial SC sources have emerged [16]. Even so, the generation of broadband SC with high brightness, high spatiotemporal coherence, and good maneuverability, is still highly desired.

The SC generation from femtosecond laser filamentation has attracted great interest due to its many advantages, including high pulse energy, high brightness, high spatiotemporal coherence, and so on [17]. In recent years, numerous studies have been performed to optimize filamentation and SC, in order to achieve high-power, ultra-broadband, and controllable SC spectrum. For example, in our previous work, we have generated a filament array in fused silica by using microlens array as the focal element and obtained a high power density SC with $\mathrm{mW} / \mathrm{nm}$ level in the visible range [18]. Lu et al. obtained an intense femtosecond SC by placing several thin fused silica plates at or near the focus 
of a high-power laser pulse. The generated SC covers from $450 \mathrm{~nm}$ to $980 \mathrm{~nm}$ with a conversion efficiency as high as 54\% [19]. Subsequently, He et al. improved the efficiency to $85 \%$ by placing more plates (seven plates at Brewster's angle), and further obtained a few-cycle pulse with a pulse duration of $5.4 \mathrm{fs}$ by using the chirped mirrors compression [20]. More recently, utilizing $1030 \mathrm{~nm} 170 \mathrm{fs}$ pulses and a two-stage multiple-plate arrangement, Lu et al. generated the SC spanning from $570 \mathrm{~nm}$ to $1300 \mathrm{~nm}$ and obtained a 3.21 fs sub-single-cycle pulse after dispersion compensation [21]. Furthermore, in the light of high power single-cycle pulse application, such as Zeptosecond-Exawatt physics, Mourou et al. theoretically demonstrated a thin film compressor that has the potential to compress a high energy pulse in a pulse as short as one optical cycle at $0.8 \mu \mathrm{m}$, producing true ultrarelativistic $\lambda^{3}$ pulses [22]. Farinella et al. experimentally demonstrated the focusability of high-power laser pulses after self-phase modulation in thin films at transport intensities $\left(\sim 1 \mathrm{TW} / \mathrm{cm}^{2}\right)$ for petawatt laser systems [23,24]. Moreover, a multi-octave SC with a wide wavelength coverage has been obtained via filamentation generation by the near- and mid-infrared ultrashort-pulse laser in the range of anomalous group velocity dispersion of wide bandgap dielectric media, such as YAG, sapphire, and fluoride crystal, or in various types of glasses [17]. For example, an ultra-broadband SC with a range from $290 \mathrm{~nm}$ to $4.3 \mu \mathrm{m}$ was generated in LiF crystal by the femtosecond laser pulses with a central wavelength of $2.3 \mu \mathrm{m}$ [25]. Therefore, the SC generated from femtosecond filamentation provides an efficient broadband light source for a variety of applications, such as SC cavity ring-down spectroscopy (SC-CRDS) [26,27], remote sensing [28], and generation of few-cycle pulses [20-24,29].

On the other hand, the modulation of SC spectrum is beneficial to many applications. To this end, several approaches have been proposed, especially to enhance the blue-side spectral extension of the SC. For instance, by introducing a positive or negative chirp into the femtosecond laser pulse, a strong and sharp spectral peak in the blue side of SC is generated from the filamentation in birefringent crystal (e.g., calcite), and the spectral peak is tunable by modulating the pulse chirp and energy [30]. Using an acousto-optic programmable dispersive filter to introduce second- and third-order phase distortions into the laser pulse, SC is obtained with controllable spectral bandwidth and shape [31]. More recently, Zhdanova et al. realized the broadening control in the visible region of the SC by using the spatial beam shaping technique [32]. Additionally, by using the input pulse with shaped phase and amplitude, the energy conversion efficiency is significantly increased within the specified bandwidths of SC [33]. Thompson et al. experimentally and theoretically demonstrated the intensity enhancement of the spectrum of femtosecond filamentation in water by applying a phase modulation into a specified frequency range [34]. Recently, Chen et al. presented a systematical investigation on the laser polarization effect on the SC generation from femtosecond laser filamentation in air. They found that the circular polarization filamenting pulse is particularly favorable for the intensity enhancement of the SC generation in the short wavelength range, and the linear polarization pulse is favorable for the spectral broadening [35]. The above-mentioned methods have achieved good results to control the characteristics of SC, such as the spectral range, the cutoff wavelength, and the intensity. However, the controllable local spectral intensity enhancement of the SC from filamentation has not yet been studied, to the best of our knowledge. In this paper, we study the control of the SC spectrum in the fused silica by the shaped femtosecond laser through feedback iteration optimization. The results show that the spectral hump in the visible region of SC can be controlled and arbitrarily shifted.

\section{Experiment Methods}

Figure 1 shows the schematic of the experiment setup. The laser is generated from a commercial Ti: Sapphire femtosecond laser system (Spitfire, Spectra-Physics) which delivers pulses at a center wavelength of $800 \mathrm{~nm}$ with a duration of $45 \mathrm{fs}$, and a repetition rate of $1 \mathrm{kHz}$. The output spectral bandwidth is about $50 \mathrm{~nm}$. The beam diameter is reduced to $\sim 7 \mathrm{~mm}$ via a telescope system. The pulse shaper design is similar to the 
report by Weiner [36], which consists of a $4 \mathrm{f}$ zero-dispersion pulse compressor and a $792 \times 600$ element pixel liquid crystal spatial light modulator (SLM, X10468-02, Hamamatsu) placed in the Fourier plane of the $4 \mathrm{f}$ system, shown in the dotted box of Figure 1. Here, a diffraction grating of 1200 lines $/ \mathrm{mm}$ and a cylindrical lens with a focal length of $300 \mathrm{~mm}$ are employed. The SLM can introduce phase modulation to the spectral components from $750 \mathrm{~nm}$ to $850 \mathrm{~nm}$, and achieve phase modulation up to $2 \pi$ radians over the readout wavelength range. Therefore, the pulse shaper has a spectral resolution of $\sim 0.13 \mathrm{~nm}$. The shaped laser pulse with different profiles can be obtained by applying different phase masks to the SLM. Thereafter, the shaped laser pulses are focused by a plano-convex lens L1 into a fused silica block to form the filament. In this experiment, the input laser energy is $\sim 6 \mu \mathrm{J}$ which is attenuated by a combination of neutral density filters and beam splitters. The focusing length of the L1 is $250 \mathrm{~mm}$, and the distance between L1 and the front surface of the fused silica is $245 \mathrm{~mm}$. The size of fused silica is $50 \times 30 \times 30 \mathrm{~mm}^{3}$. Based on the parameters of the shaping pulse, it can be estimated that the peak intensity at the input of fused silica is $\sim 4 \times 10^{10} \mathrm{~W} / \mathrm{cm}^{2}$. The SC generated from filamentation in the fused silica is collected by a plano-convex lens $\mathrm{L} 2(\mathrm{f}=100 \mathrm{~mm})$ into an integrating sphere (IS) connected to a spectrometer (USB 4000, Ocean Optics Inc.). A dichroic mirror $(\mathrm{R}>98 \% @ \sim 350 \mathrm{~nm}-\sim 670 \mathrm{~nm}$ ) is put before the IS to block the fundamental light, and the integration time of the spectrometer is $15 \mathrm{~s}$.

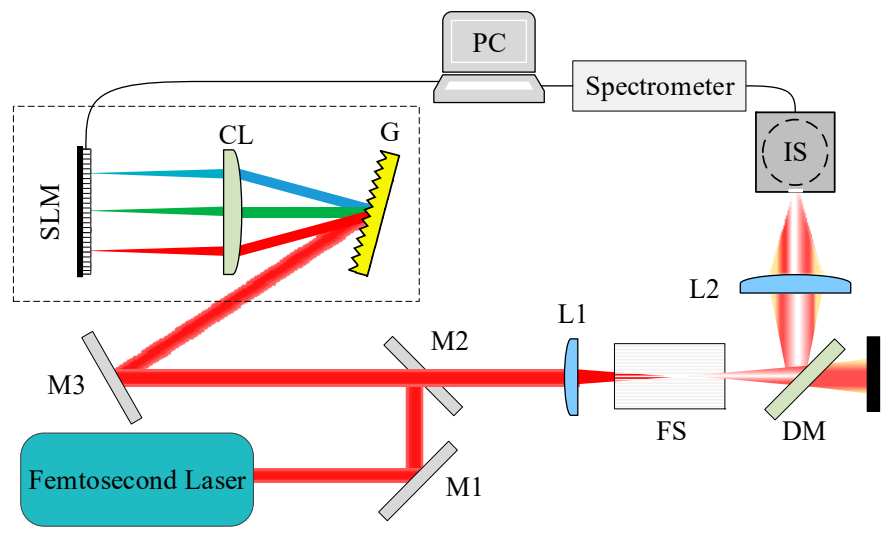

Figure 1. The schematic of the experimental setup. M: $800 \mathrm{~nm}$ high reflective mirror. G: grating. CL: cylindrical lens. SLM: spatial light modulator. L: plano-convex lens. FS: fused silica. DM: dichroic mirror. IS: integrating sphere.

According to the experiment settings, we design a feedback control program based on the genetic algorithm (GA), in which the spectral intensity of SC is set to be the feedback signal of the GA. In the experiment, we set 50 phase arrays for each generation, and the phase arrays of the initial iteration are generated randomly. To reduce the search space of GA, every 8 consecutive pixels are combined in sets and assigned the same value of phase retardance, resulting in 99 effective pixels. For each iteration, the SC generation by the shaped laser pulse corresponding to each phase array is evaluated via the fitness value given by the GA. After that, the 50 compensation phase arrays are sequenced based on the evaluation results. The better 20 of them are selected and used to create the new 50 phase arrays for the next generation by the methods of crossover and mutation. The program will exit as soon as the fitness function is satisfied.

\section{Results and Discussion}

Using the experimental setups and the control programs, we study the control of the SC spectrum generated from filamentation in fused silica by shaped femtosecond laser pulses. To start with, we define the fitness function as the spectral intensity of $600 \mathrm{~nm}$ in the SC. Through the feedback iteration optimization, the enhancement of spectral intensity at $600 \mathrm{~nm}$ is realized. The SC spectra for different generations are shown in Figure 2. Note 
that since the dichroic mirror blocks the fundamental light, only the spectral region less than $670 \mathrm{~nm}$ is left. Correspondingly, the phase masks for several typical generations are shown in Figure 3. The mask is a grayscale image corresponding to the phase change from 0 to $2 \pi$ on SLM. From Figure 2, we can find that the SC spectral intensity around $600 \mathrm{~nm}$ is enhanced significantly with the increase in the generation number. Especially, in the profile of SC, around $600 \mathrm{~nm}$ is changed dramatically. Since the 4th generation, an obvious spectral hump appears near $600 \mathrm{~nm}$. From the 9th generation, the intensity of the spectral hump increases rapidly and reaches the maximum intensity at the 12th generation. The intensity of the hump at $600 \mathrm{~nm}$ increases first and decreases due to the hump shift, and then increases rapidly. Finally, the position of the hump is stabilized at $600 \mathrm{~nm}$ with a small deviation, which indicates that the spectral hump at $600 \mathrm{~nm}$ is obtained through the feedback pulse shaping control.

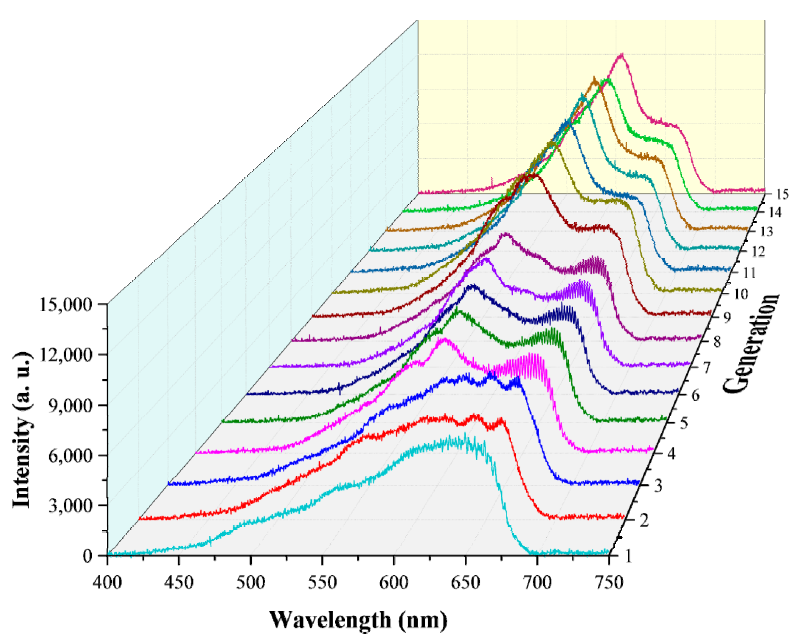

Figure 2. The SC spectra for different generations.

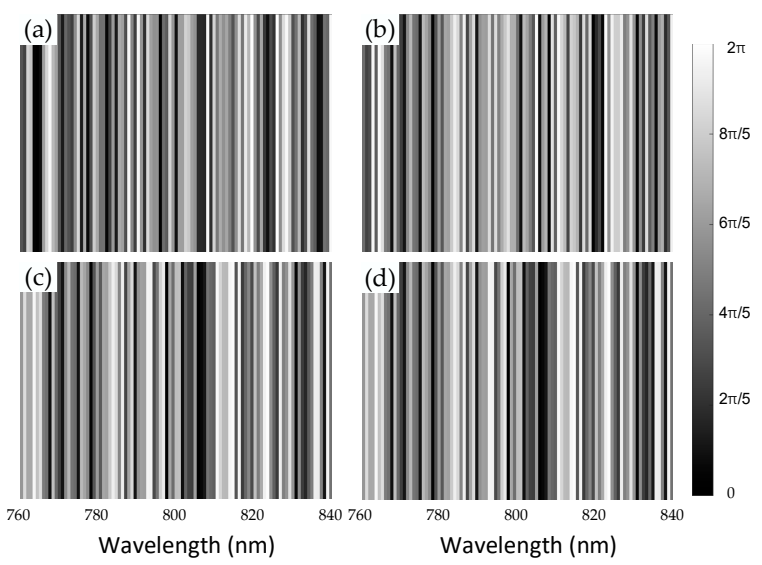

Figure 3. The optimal phase masks for the (a) 1st, (b) 5th, (c) 10th, and (d) 15th generation (corresponding to Figure 2), respectively.

In order to clearly show the evolution of the spectral hump generated by the shaped femtosecond pulses with the increase in the feedback iterations, the spectral intensity at $600 \mathrm{~nm}$ in each generation in Figure 2 is extracted and plotted in Figure 4a. We can see that the spectral intensity at $600 \mathrm{~nm}$ increases slowly with the number of iterations initially, and then decreases from the 5th generation and reaches the minimum after two generations. Thereafter, the spectral intensity dramatically increases and soon reaches a maximum at the 12th generation and is stabilized gradually. In the same way, we extract the position of the spectral hump of the SC for each generation and plot it in Figure 4b. It should be noted 
that in the first three generations no obvious spectral hump appears in the SC spectrum near the wavelength of $600 \mathrm{~nm}$, so the spectral position of these three generations is not shown in Figure $4 \mathrm{~b}$. From this figure, we can see that the hump position deviates from the $600 \mathrm{~nm}$ wavelength first and then approaches, which corresponds to the spectral intensity fluctuation shown in Figure 4a. Eventually, the hump position is relatively stable at about $600 \mathrm{~nm}$. As a result, we obtain a spectral hump in the blue-side of the SC by using shaped laser pulses and realize the stabilization of the spectral position of the hump to a preset wavelength of $600 \mathrm{~nm}$.
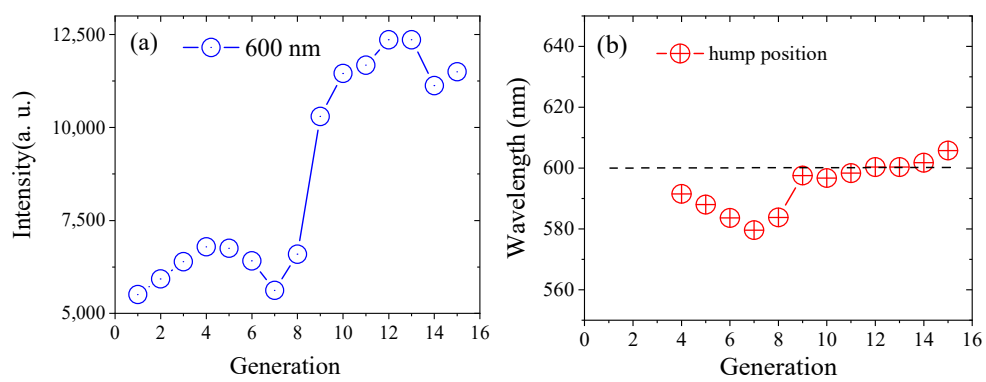

Figure 4. The spectral intensity at $600 \mathrm{~nm}$ (a) and the hump position of the SC (b) as a function of generation.

To illustrate the universality of the spectral hump modulation of SC generated from shaped laser pulse filamentation through feedback iteration, we further investigate the spectral control at other wavelengths by the same method. By changing the fitness function to the spectral intensity of $510 \mathrm{~nm}$, a similar evolution of SC to that shown in the Figure 2 is obtained. After two iterations, the spectral enhancement occurs at about $510 \mathrm{~nm}$. With the further increase in iteration, the spectral hump position oscillates around $510 \mathrm{~nm}$, and the intensity of the hump increases. After nine iterations, the spectral hump is stabilized at $510 \mathrm{~nm}$, and the hump intensity no longer changes significantly with the increase in iteration. Further changing the fitness function of GA to the spectral intensity of other wavelengths ( $560 \mathrm{~nm}$ and $655 \mathrm{~nm}$, respectively), the formation of the spectral humps at the preset spectral positions in the visible region of SC is realized. The four typical results of the spectra hump modulation by the shaped femtosecond pulses are shown in Figure 5. The hump positions are well stabilized at $510 \mathrm{~nm}, 560 \mathrm{~nm}, 600 \mathrm{~nm}, 655 \mathrm{~nm}$, respectively.

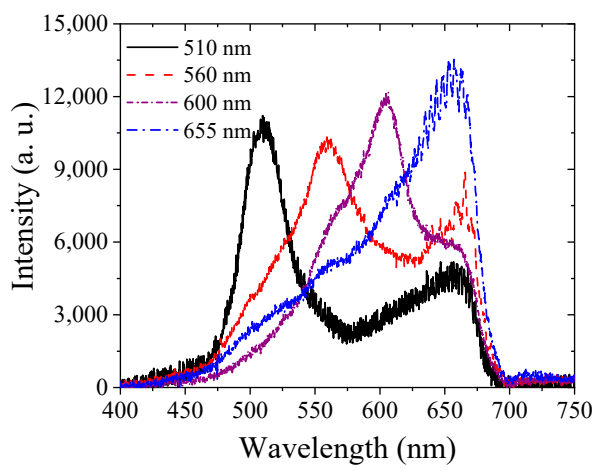

Figure 5. The spectral hump in the SC is generated by shaped femtosecond pulses with different fitness functions.

In Figure 6, we plot the temporal envelope of shaped femtosecond pulses used to generate the SC shown in Figure 5. Because the pulse envelope can not be measured directly under our present experimental condition, we calculate the temporal envelope of shaped femtosecond pulses based on the spectrum of the initial laser pulse and the phase introduced by SLM [37]. As can be seen from Figure 6, the pulse width reaches the order of 
picosecond after shaping, and the envelope becomes more complicated and is no longer a Gaussian distribution.

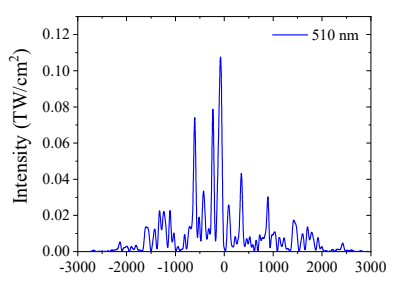

$\mathrm{t}(\mathrm{fs})$

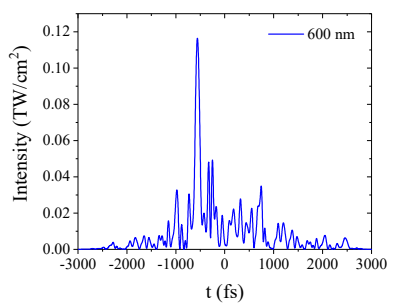

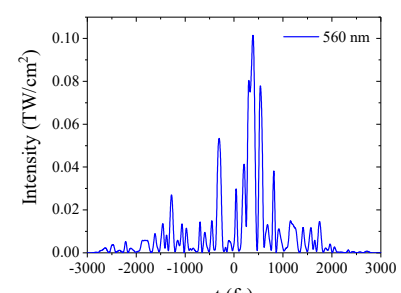

$\mathrm{t}(\mathrm{fs})$

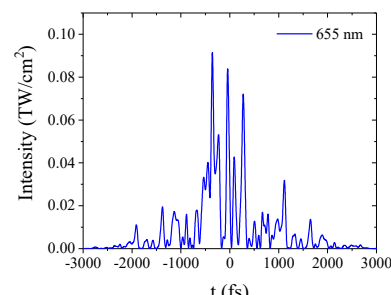

$\mathrm{t}(\mathrm{fs})$

Figure 6. The shaped femtosecond pulses by the pulse shaper for filamentation and SC generation for the cases shown in Figure 5.

The self-phase modulation (SPM) is regarded as the main mechanism causing the spectral broadening of the intense femtosecond laser pulse. Our previous studies revealed that the pulse shape plays a dominant role in the filamentation and SC generation due to the SPM and electron generation [38,39]. More complicated pulse shapes will induce steeper ascending and descending edges in the laser pulse, which can lead stronger blueside spectral extension [39]. In our experiment, the pulse is modulated to an extremely complicated pulse with many temporal peaks, as shown in Figure 6. These pulse peaks will induce a very strong SPM effect and very fast electron generation during the process of filamentation. As a consequence, the pulse spectrum will have a huge extension, especially to the blue-side. Therefore, it is understandable that the SC spectral intensity can be enhanced by using pulse shaping technology and feedback iteration. However, various nonlinear effects, including SPM, electron generation, self-steepening, high-order Kerr effect, and so on, are involved in the filamentation and SC generation, especially for the ultrashort pulses with modulated shapes, which deserves further study.

\section{Conclusions}

Based on the feedback optimal control, we experimentally study the spectral modulation in the visible region of SC generated from the shaped laser pulse filamentation in fused silica and realize the intensity enhancement at selected wavelengths. The spectral hump in the visible region of SC can be formed at desired wavelength positions. The control of the spectral hump of the SC could improve the abilities of the SC applications where the spectral power of different spectral ranges needs to be flexibly enhanced. For example, in the SC-CRDS, for different absorbers, the light source should have high enough spectral power at different wavelength bandwidths [27].

Author Contributions: Conceptualization, Z.H.; Data curation, J.C. and D.L.; Investigation, J.C.; Methodology, J.C., L.Z. and Z.H.; Software, J.C. and T.X.; Writing-original draft, J.C.; Writingreview \& editing, T.X. and Z.H. All authors have read and agreed to the published version of the manuscript.

Funding: This research was funded by the National Natural Science Foundation of China (Grant numbers: $12074228,11774038,11874056,11474039)$, the Taishan scholar project of Shandong Province (Grant number: tsqn201812043), the Innovation Group of Jinan (Grant number: 2020GXRC039), and the Youth Innovation Promotion Association of the Chinese Academy of Sciences (Grant number: 2014143). 
Data Availability Statement: The data that support the findings of this study are available from thecorresponding author upon reasonable request.

Conflicts of Interest: The authors declare no conflict of interest.

\section{References}

1. Alfano, R.R.; Shapiro, S.L. Emission in the region 4000 to $7000 \AA$ Aia four-photon coupling in glass. Phys. Rev. Lett. 1970, 24, 584-587. [CrossRef]

2. Alfano, R.R.; Shapiro, S.L. Observation of Self-phase modulation and small-scale filaments in crystals and glasses. Phys. Rev. Lett. 1970, 24, 592-594. [CrossRef]

3. Riedle, E.; Bradler, M.; Wenninger, M.; Sailer, C.F.; Pugliesi, I. Electronic transient spectroscopy from the deep UV to the NIR: Unambiguous disentanglement of complex processes. Faraday Discuss 2013, 163, 139-158. [CrossRef]

4. Petersen, C.R.; Moselund, P.M.; Huot, L.; Hooper, L.; Bang, O. Towards a table-top synchrotron based on supercontinuum generation. Infrared Phys. Tech. 2018, 91, 182-186. [CrossRef]

5. Dupont, S.; Petersen, C.; Thøgersen, J.; Agger, C.; Bang, O.; Keiding, S.R. IR microscopy utilizing intense supercontinuum light source. Opt. Express 2012, 20, 4887-4892. [CrossRef]

6. Colley, C.S.; Hebden, J.C.; Delpy, D.T.; Cambrey, A.D.; Brown, R.A.; Zibik, E.A.; Ng, W.H.; Wilson, L.R.; Cockburn, J.W. Mid-infrared optical coherence tomography. Rev. Sci. Instrum. 2007, 78, 123108. [CrossRef]

7. Petersen, C.R.; Prtljaga, N.; Farries, M.; Ward, J.; Napier, B.; Lloyd, G.R.; Nallala, J.; Stone, N.; Bang, O. Mid-infrared multispectral tissue imaging using a chalcogenide fiber supercontinuum source. Opt. Lett. 2018, 43, 999-1002. [CrossRef]

8. Kaminski, C.F.; Watt, R.S.; Elder, A.D.; Frank, J.H.; Hult, J. Supercontinuum radiation for applications in chemical sensing and microscopy. Appl. Phys. B 2008, 92,367-378. [CrossRef]

9. Alfano, R.R. The Supercontinuum Laser Source: Fundamentals with Updated References; Springer: New York, NY, USA, 2006.

10. Dudley, J.M.; Genty, G.; Coen, S. Supercontinuum generation in photonic crystal fiber. Rev. Mod. Phys. 2006, 78, 1135-1184. [CrossRef]

11. Price, J.H.V.; Feng, X.; Heidt, A.M.; Brambilla, G.; Horak, P.; Poletti, F.; Ponzo, G.; Petropoulos, P.; Petrovich, M.; Shi, J.; et al. Supercontinuum generation in non-silica fibers. Opt. Fiber Technol. 2012, 18, 327-344. [CrossRef]

12. Dudley, J.M.; Taylor, J.R. Supercontinuum Generation in Optical Fibers; Cambridge University Press: Cambridge, UK, 2010.

13. Diouf, M.; Salem, A.B.; Cherif, R.; Saghaei, H.; Wague, A. Super-flat coherent supercontinuum source in As38.8Se61.2 chalcogenide photonic crystal fiber with all-normal dispersion engineering at a very low input energy. Appl. Opt. 2017, 56, 163-169. [CrossRef] [PubMed]

14. Adamu, A.I.; Habib, M.S.; Petersen, C.R.; Lopez, J.E.A.; Zhou, B.; Schülzgen, A.; Bache, M.; Amezcua-Correa, R.; Bang, O.; Markos, C. Deep-UV to Mid-IR supercontinuum generation driven by Mid-IR ultrashort pulses in a gas-filled hollow-core fiber. Sci. Rep. 2019, 9, 4446. [CrossRef]

15. Petersen, C.R.; Møller, U.; Kubat, I.; Zhou, B.; Dupont, S.; Ramsay, J.; Benson, T.; Sujecki, S.; Abdel-Moneim, N.; Tang, Z.; et al. Mid-infrared supercontinuum covering the 1.4-13.3 $\mu \mathrm{m}$ molecular fingerprint region using ultra-high NA chalcogenide step-index fibre. Nat. Photonics 2014, 8, 830. [CrossRef]

16. Labruyère, A.; Tonello, A.; Couderc, V.; Huss, G.; Leproux, P. Compact supercontinuum sources and their biomedical applications. Opt. Fiber Technol. 2012, 18, 375-378. [CrossRef]

17. Dubietis, A.; Tamošauskas, G.; Šuminas, R.; Jukna, V.; Couairon, A. Ultrafast supercontinuum generation in bulk condensed media (Invited Review). Lith. J. Phys. 2017, 57, 113-157. [CrossRef]

18. Camino, A.; Hao, Z.; Liu, X.; Lin, J. High spectral power femtosecond supercontinuum source by use of microlens array. Opt. Lett. 2014, 39, 747-750. [CrossRef]

19. Lu, C.-H.; Tsou, Y.-J.; Chen, H.-Y.; Chen, B.-H.; Cheng, Y.-C.; Yang, S.-D.; Chen, M.-C.; Hsu, C.-C.; Kung, A.H. Generation of intense supercontinuum in condensed media. Optica 2014, 1, 400-406. [CrossRef]

20. He, P.; Liu, Y.; Zhao, K.; Teng, H.; He, X.; Huang, P.; Huang, H.; Zhong, S.; Jiang, Y.; Fang, S.; et al. High-efficiency supercontinuum generation in solid thin plates at 0.1TW level. Opt. Lett. 2017, 42, 474-477. [CrossRef]

21. Lu, C.-H.; Wu, W.-H.; Kuo, S.-H.; Guo, J.-Y.; Chen, M.-C.; Yang, S.-D.; Kung, A.H. Greater than 50 times compression of 1030 nm $\mathrm{Yb}: \mathrm{KGW}$ laser pulses to single-cycle duration. Opt. Express 2019, 27, 15638-15648. [CrossRef]

22. Mourou, G.; Mironov, S.; Khazanov, E.; Sergeev, A. Single cycle thin film compressor opening the door to Zeptosecond-Exawatt physics. Eur. Phys. J. Spec. Top. 2014, 223, 1181-1188. [CrossRef]

23. Anashkina, E.A.; Ginzburg, V.N.; Kochetkov, A.A.; Yakovlev, I.V.; Kim, A.V.; Khazanov, E.A. Single-shot laser pulse reconstruction based on self-phase modulated spectra measurements. Sci. Rep. 2016, 6, 33749. [CrossRef]

24. Farinella, D.M.; Wheeler, J.; Hussein, A.E.; Nees, J.; Stanfield, M.; Beier, N.; Ma, Y.; Cojocaru, G.; Ungureanu, R.; Pittman, M.; et al. Focusability of laser pulses at petawatt transport intensities in thin-film compression. J. Opt. Soc. Am. B 2019, 36, A28. [CrossRef]

25. Garejev, N.; Tamošauskas, G.; Dubietis, A. Comparative study of multioctave supercontinuum generation in fused silica, YAG, and $\mathrm{LiF}$ in the range of anomalous group velocity dispersion. J. Opt. Soc. Am. B 2017, 34, 88-94. [CrossRef]

26. Stelmaszczyk, K.; Fechner, M.; Rohwetter, P.; Queißer, M.; Czyżewski, A.; Stacewicz, T.; Wöste, L. Towards supercontinuum cavity ring-down spectroscopy. Appl. Phys. B 2009, 94, 369-373. [CrossRef] 
27. Camino, A.; Li, S.W.; Hao, Z.Q.; Lin, J.Q. Spectroscopic determination of NO2, NO3, and O3 temporal evolution induced by femtosecond filamentation in air. Appl. Phys. Lett. 2015, 106, 021105. [CrossRef]

28. Hakala, T.; Suomalainen, J.; Kaasalainen, S.; Chen, Y. “Full waveform hyperspectral LiDAR for terrestrial laser scanning. Opt. Express 2012, 20, 7119-7127. [CrossRef]

29. Budriūnas, R.; Stanislauskas, T.; Adamonis, J.; Aleknavičius, A.; Veitas, G.; Gadonas, D.; Balickas, S.; Michailovas, A.; Varanavičius, A. $53 \mathrm{~W}$ average power CEP-stabilized OPCPA system delivering $5.5 \mathrm{TW}$ few cycle pulses at $1 \mathrm{kHz}$ repetition rate. Opt. Express 2017, 25, 5797-5806. [CrossRef]

30. Kartazaev, V.; Alfano, R.R. Supercontinuum generated in calcite with chirped femtosecond pulses. Opt. Lett. 2007, 32, 3293-3295. [CrossRef]

31. Dharmadhikari, J.A.; Dharmadhikari, A.K.; Dota, K.; Mathur, D. Influencing supercontinuum generation by phase distorting an ultrashort laser pulse. Opt. Lett. 2015, 40, 241-244. [CrossRef]

32. Zhdanova, A.A.; Shen, Y.; Thompson, J.V.; Scully, M.O.; Yakovlev, V.V.; Sokolov, A.V. Controlled supercontinua via spatial beam shaping. J. Mod. Opt. 2018, 65, 1332-1335. [CrossRef]

33. Schumacher, D. Controlling continuum generation. Opt. Lett. 2002, 27, 451-453. [CrossRef]

34. Thompson, J.V.; Zhokhov, P.A.; Springer, M.M.; Traverso, A.J.; Yakovlev, V.V.; Zheltikov, A.M.; Sokolov, A.V.; Scully, M.O. Amplitude concentration in a phase-modulated spectrum due to femtosecond filamentation. Sci. Rep. 2017, 7, 43367. [CrossRef] [PubMed]

35. Chen, N.; Wang, T.-J.; Zhu, Z.; Guo, H.; Liu, Y.; Yin, F.; Sun, H.; Leng, Y.; Li, R. Laser ellipticity-dependent supercontinuum generation by femtosecond laser filamentation in air. Opt. Lett. 2020, 45, 4444-4447. [CrossRef] [PubMed]

36. Weiner, A.M. Femtosecond pulse shaping using spatial light modulators. Rev. Sci. Instrum. 2000, 71, 1929-1960. [CrossRef]

37. Chen, A.; Li, S.; Qi, H.; Jiang, Y.; Hu, Z.; Huang, X.; Jin, M. Elongation of plasma channel generated by temporally shaped femtosecond laser pulse. Opt. Commun. 2017, 383, 144-147. [CrossRef]

38. Zhan, L.; Xu, M.; Xi, T.; Hao, Z. Contributions of leading and tailing pulse edges to filamentation and supercontinuum generation of femtosecond pulses in air. Phys. Plasmas 2018, 25, 103102. [CrossRef]

39. Xu, M.; Zhan, L.; Xi, T.; Hao, Z. Supercontinuum generation by femtosecond flat-top laser pulses in fused silica. J. Opt. Soc. Am. B 2019, 36, G6. [CrossRef] 